Pryscilla Batista Leite $1 *$ (1) Andressa da Silva Coelho ${ }^{1}$ Maria Ivanda Rio ${ }^{1}$

Nathalia Almeida Freire ${ }^{2}$ Maria Eliza Barbosa Ramos ${ }^{2}$ Monica Simoes Israel ${ }^{2}$

\section{Oral manifestations of covid-19: review of the literature}

\begin{abstract}
:
COVID-19 is caused by the SARS-CoV-2 virus and proved to be a challenge since there was little knowledge about this disease. Several viral infections can affect the oral cavity and because of this is fundamental the study of this disease and whether oral manifestations could appear in an oral cavity. This work aims to review the literature on what is in the scientific articles of the oral manifestations of COVID-19 since the recognition of the existence of the pandemic.
\end{abstract}

Keywords: Coronavirus, Oral Health, Coronavirus Infections, Oral Manifestations

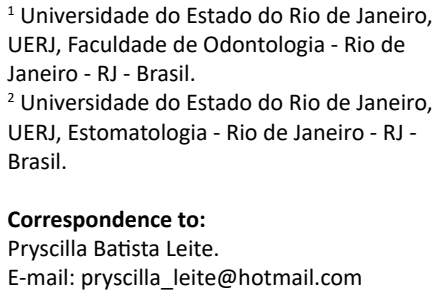

Article received on March 23, 2021. Article accepted on June 17, 2021.

DOI: $10.5935 / 2525-5711.20210014$ 


\section{INTRODUCTION}

The COVID-19 infection consists of a viral respiratory disease caused by SARS-CoV-2, which is transmitted through aerosols, droplets and, contaminated objects $^{1}$. This virus enters the target cells through ACE2 receptors, spreading to other regions of the body ${ }^{2,3}$.

The most common symptoms found in patients with COVID-19 are fever, cough, and headache. In the most severe cases, the virus may be responsible for respiratory distress syndrome or even lead to death4. There are several reports in the literature about the possible oral manifestations of the disease, such as soft tissue ulcerations, vesiculobullous diseases, changes in the salivary and neurological glands $s^{3,4}$.

As dentists were considered high-risk health professionals due to exposure to dental procedures, it is essential to know the possible lesions of COVID-19 both for the prevention and early detection of oral manifestations.

This study aims to present the oral manifestations of COVID-19 seen in the literature to help in the early diagnosis and control of the transmission.

\section{REVIEW OF LITERATURE}

Galván Casas et al.5 described the cutaneous manifestations of COVID-19 in a series of 375 cases and documented for the first time the oral manifestations that occurred during the course of the disease in an atlas with 150 cases. Three cases were found with intraoral findings ranging from ulcers in the palate mucosa to erythema located on the palate and gingival margin ${ }^{1,5}$.

The oral manifestations found in the oral cavity of patients with COVID-19 are ulcers, blisters, erythematous plaques, macules, desquamative gingivitis, and herpetic stomatitis ${ }^{3,6,7,8}$.

Oral aphthous ulcerations have been reported both in immunosuppressed elderly patients and in young people. The healing process of these ulcers occurred when patients were treated for the disease $e^{3,6}$.

One report presented an ulcer that healed completely in 10 days, however, on the third day of symptomatology, there was the development of a flat lesion in the thumb of the foot, which raises the hypothesis of manifestation of viral disease ${ }^{9}$.

There are chronic diseases that are associated with poor prognosis in patients with SARS-CoV-2, so herpetic lesions on the palate may be associated with emotional stress ${ }^{6,10}$. There are questions as to whether oral manifestations during the disease may be secondary to compromised systemic health or treatment with systemic steroids and topics to which patients are submitted during the course of infection.

Examples of this are seen in immunological conditions such as pemphigus, lichen planus, and pemphigoide. In addition, changes in the oral microbiota may also occur that may cause hypersalivation or xerostomia, as well as fungal, bacterial infections (such as Necrotizing Ulcerative Gingivitis) and ulcerations. These findings were observed in patients with COVID-196,8,10,11.

It is known that viral infections may present manifestations in the oral cavity, such as Dengue, Ebola virus, Hand-foot-mouth disease, Herpes Simplex Virus infection, HIV infection, Infections caused by Epstein-Barr virus, and Measles. They can arise from maculopapular infections to painful ulcers and blisters. Because of this, the study on the possible oral involvement of SARS-CoV-2 infection is extremely important ${ }^{12,13}$.

A case of vesiculobullous lesion on the lip with erythematous halo has been reported in the literature in a patient with COVID-19, in whom he was treated with Dexamethasone and had remission of the lesion from the eighth day. A biopsy was performed and a moderate lymphocytic infiltrate and microvascular thrombosis were seen on histopathological examination. The result for Herpes-simplex virus (HSV-1), HSV-2, HSV-3, and cytomegalovirus was negative ${ }^{13}$.

Perilabial lesions, characteristics of recurrent herpetic stomatitis with a course similar to that of patients without a diagnosis of COVID-19 in a patient with mild symptoms, have been described in the literature by Kitakawa et $\mathrm{al}^{14}$.

The evolution of Herpes Zoster has been reported in the literature ${ }^{15,44}$. In one of the cases, the patient presented facial involvement on the left side and intraoral lesions of an oligosymptomatic infection of COVID- $19^{15}$. Lesions similar to those occurring in Herpes Simplex and multiform erythema affecting both keratinized and non-keratinized tissue were found in three patients according to Sinadinos et al. ${ }^{16}$ Among the three, two had suspected COVID-19 and one had a positive result, with lesions similar to multiform erythema3,16.

In patients diagnosed with multiform erythema during hospitalization, skin lesions were found that started as erythematous papules and progressed to erythematous violaceous patches with a dark center and a pseudovesicle. These patients were submitted to oral cavity examination, which revealed macules and petechiae in the palate ${ }^{17}$. 
Histological examination of these lesions revealed mild to moderate spongiosis in the epidermis, dermis with dilated vessels and the high number of neutrophils, extravasation of red blood cells, and perivascular and interstitial lymphocytic infiltration ${ }^{17}$.

Studies have shown that the entrance door of SARS-CoV-2 is through ACE-2, which is present in the mucous membranes of the oral cavity. There is a high expression of ACE-2 in epithelial cells, especially in the tongue, gums, and salivary glands 19. The viral load of this region is related to the severity of COVID-19 $9^{13,21}$.

This increased expression in salivary glands is sustained by the high amount of salivary samples positive for SARS-CoV-2. This can cause glandular disorders, such as acute parotitis, and maybe the first sign of COVID-19 infection that can affect salivary consistency in terms of increased serous secretion and enzymatic content ${ }^{19}$.

Another possible early manifestation of COVID-19 associated with salivary glands, according to the studies, would be non-suppurative acute sialadenitis, which according to Chern et al.2 1 may affect the parotid gland and submandibular gland in patients with positive results for COVID-19 $9^{21}$.

Some studies have also reported that Squamous Cell Carcinoma could be an oral manifestation of COVID-19, since overexpression of ACE-2 and its receptors are related to several aspects of oral carcinogenesis, according to the literature20,23,24,25. Also, smoking is investigated as a consistent risk factor for Squamous Cell Carcinoma, increasing ACE-2 expression.

The prolonged duration of viral load of SARS-CoV-2 may be associated with poor hygiene of the oral cavity, which may have an impact on the hospitalization of patients ${ }^{27}$.

A study conducted at the Yage School of Medicine with 44 patients concluded that salivary samples were more sensitive and had greater consistency throughout the disease ${ }^{27}$.

Regarding salivary alterations, hypersalivation and hyposalivation were described in the literature in patients hospitalized by COVID-19 in the severe stage, who required intubation, tracheostomy, and external ventilation, being subject to hyposalivation ${ }^{10}$.

Hypersalivation was reported as secondary to angular cheilitis after patients tested positive and presented pain in both labial commissures ${ }^{28}$.

Concerning the loss of taste, xerostomia, burning sensation in the oral cavity, studies show the onset of these symptoms before other typical symptoms of COVID, as well as after hospitalization ${ }^{29,30}$.

However, about xerostomia, it has not yet been proven whether it can be caused by the use of medicines or an active virus infection ${ }^{30}$.

The impact of the dysbiotic oral microbiota, as an entrance door to SARS-CoV-2, is still questioned as to the correlation between oral dysbiosis and disease severity in infected patients ${ }^{18}$.

One more point related to oral manifestations associated with COVID-19 that deserves attention is the probability of the development of the periodontal disease. Chronic systemic inflammation associated with periodontitis may present a higher risk of developing COVID-19 more severely ${ }^{31}$.

Besides periodontitis was also related to increased adverse outcomes in patients, such as increased mortality, need for ICU hospitalization and assisted ventilation $^{34}$. Marouf et al. also reported that patients with periodontitis had an increase in leukocytes and higher serum C-reative protein levels when compared to people without this chronic disease, which could show that the relationship between periodontitis and COVID-19 is linked to systemic inflammation ${ }^{32}$.

In addition to the manifestations described, studies have demonstrated findings such as transient lingual papillitis, glossitis with lateral indentations, aphthous stomatitis, glossitis with despapilation and mucositis, as well as burning sensation and dysgeusia ${ }^{33}$.

According to the American Academy of Otorhinolaryngology-Head and Neck Surgery (AAO-HNS) when a patient has anosmia, hyposmia, and dysgeusia in the absence of rhinorrhea or rhinitis, should be paid care to the possibility of SARS-CoV-2 infection. ${ }^{34,35,36}$

According to a study by Biadsee et al. who verified a series of cases of 140 patients who participated in an online questionnaire about the most common symptoms of viral diseases such as olfactory and taste disorders, xerostomia, and orofacial pain. Such symptoms were more common in women ${ }^{34,36}$.

As for neurological manifestations, a case of vestibular neuritis was reported, which is a viral or post-viral inflammatory lesion that affects the VIII cranial nerve ${ }^{37}$.

Dentistry has shown a fundamental role in the evolution of this viral infection in patients with cardiovascular diseases and COVID-19, an example of this is in controlling the development of bacteremia in the oral cavity ${ }^{38}$. 
Certainly, intraoral examination in patients with the positive result for COVID-19 or who are suspected of this disease can assist the study on oral manifestations. This knowledge allows us to differentiate whether the lesions are characteristic of the disease, resulting from associated therapies or responses to stress resulting from the presence of the virus ${ }^{6,7}$.

\section{DISCUSSION}

There are several nonspecific manifestations of COVID-19 in the oral cavity reported in the literature, although the etiology of some of these associations has not yet been scientifically proven.

Among the findings, there is the possibility of the dysbiotic oral microbiota being an entrance door to the SARS-CoV-2. Moreover, it is also questioned whether there is a correlation between oral dysbiosis and the severity of COVID-19 in infected patients. The impact of the disease or medication is also questioned about the possibility of generating deleterious effects on saliva about flow and composition ${ }^{13,18}$.

The pharmacotherapies to which patients are submitted to the treatment of COVID-19 may also be able to cause changes in the balance of the oral microbiota, and other effects like a manifestation of gingivitis, ulcers, hypersalivation, xerostomia, fungal infections, or oral sensations of neurological basis may occur ${ }^{6,7,10}$.

It is also suggested that the state of immunosuppression caused by the SARS-CoV-2 virus may allow the appearance of lesions in the oral cavity, since cases of patients in this condition and with the positive result for COVID-19 who presented dysgeusia, anosmia, aphthous lesions, burning sensation, decreased tongue papillation, pseudomembranous candidiasis or angular cheilitis were reported ${ }^{29,32}$.

Furthermore, there are questions whether oral manifestations during the disease may be secondary to compromised systemic health or to systemic and topical steroid treatments to which patients are submitted during the course of the disease $\mathrm{e}^{7,10}$.

Considering that Angiotensin II is part of the mechanism of action of SARS-CoV-2 infection, it has been reported that several organs have a high expression, including the oral cavity. This can occur in the epithelial cells of the mucosa, affecting mainly the tongue and gums. These findings may contribute to a better understanding of oral virus infection ${ }^{25}$.

In addition to tongue and gum involvement, expression in salivary glands has also been reported and can cause acute parotitis, which may be the first sign of SARS-CoV-2 infection, also associated with intraparotid lindadenitis ${ }^{19,39}$.

Angiotensin II is capable of promoting the invasion and cell migration of Squamous Cell Carcinoma in an autocrine manner, triggering tumor interactions mediated by its receptors ${ }^{24,25,26}$. The association of ACE2 and Oral Squamous Cell Carcinoma goes beyond the high expression found in the mouth. It is important to evaluate this association since this most common malignancy of the oral cavity is the result of activation and deregulation of biological processes generated by a complex interaction between environmental factors, epigenetic alterations, and genetic variations ${ }^{2,20,29,23,24}$.

Although this seems to act as a protective mechanism against tumor progression, the harmful effects of tobacco, together with the entry of SARS-CoV-2 into epithelial cells, could be increased and contribute to cancer progression ${ }^{22,23,25}$. This pandemic should teach us several lessons and the possible relationship between Squamous Cell Carcinoma and SARS-CoV-2, which need to be further investigated ${ }^{9,22}$.

Most oral manifestations reported in the literature demonstrated pain, desquamative gingivitis, ulcers, erythema, and blisters related to SARS-CoV-2 1,6 infection. Ulcerated lesions are generally more frequent in patients diagnosed with COVID-19 positive diagnosis. The most common localization sites were the back of the tongue, hard palate, and oral mucosa, with reports of painful symptomatology. However, oral manifestations may have several clinical presentations ${ }^{3,6,40}$.

Younger patients with COVID-19 in lighter stages had aphthous ulcerations. On the other hand, immunosuppressed elderly with more severe stages of SARS-CoV-2 infection manifested ulceration with necrotic surface ${ }^{3}$.

Oral lesions may also be vesiculobullous or macular, from blisters to erythematous and petechiae lesions. Lesions like Multiform erythema have also been reported, which is the most common and associated with skin lesions ${ }^{18}$. Besides that, mucocutaneous manifestations were reported in a study published by the British Journal of Dermatology with 666 patients with COVID-19 and a slight female predominance, which can be explained by sexual dimorphism in the olfactory bulb ${ }^{33}$.

Cases of Herpes Zoster, Herpes Simplex, and multiform erythema have been reported in patients affected by the SARS-CoV-2 virus. According to the literature, the lesions presented in initial infections in patients with positive PCR. Histopathological examination showed 
moderate lymphocytic infiltrate and microvascular thrombosis ${ }^{41}$.

Typical symptoms of COVID-19 may be independent of oral manifestations of the disease. Freni $\mathrm{F}$ et al. ${ }^{30}$ suggest the appearance of symptoms in the oral cavity resulting from the impediment of salivary flow in patients infected with SARS-CoV-2.

According to what was seen in the studies, due to patients hospitalized with COVID-19 in severe stage needing intubation, tracheostomy, and external ventilation, hyposalivation was pointed out as a result of this process. As hyposalivation can reduce the patient's oral health, it can have an impact on their hospitalization ${ }^{10,26}$.

Anosmia is considered a marker for COVID-19 according to renowned international medical entities such as the British Association for Otorhinolaryngolo$\mathrm{gy}^{35}$. It is believed that there are two possible ways for the entry of the virus into the Central Nervous System (CNS): through circulation or the cribriform lamina of the ethmoid bone ${ }^{34,42}$.

A manifestation of COVID-19 in which dentists are sought is dysgeusia when the patients notice this type of alteration. Regarding oral manifestations, local oral infections and diseases of the upper respiratory tract can cause olfactory disorders, which in turn can trigger changes in taste ${ }^{34,35,36}$.

This was more frequently identified in patients who had such manifestations before dry cough, fever, and fatigue and all patients with taste disorder also had olfactory alterations ${ }^{4}$.

Also, some studies have reported neurological symptoms in patients positive for SARS-CoV-2, such as dizziness, headache, decreased consciousness, convulsions, symptoms related to cranial nerve pairs such as dysgeusia, anosmia, and vision problems ${ }^{37}$.

According to the literature, there are still no answers regarding neurological manifestations related to dentistry, as well as the correlation between oral health and bacteremia in the worsening of cardiac patients ${ }^{7,37}$.

Another point associated with the possible oral manifestations of COVID-19 that deserve attention is the probability of the development of the periodontal disease, since the pathophysiology of NETosis, a form of cell death that occurs due to chromatin decondensation through neutrophils, is also found in periodontal disease and COVID- $19^{31}$.

As the disease progresses and in more severe stages of COVID-19, may occur the "inflammatory storm". At this stage, there is an increase in the number of extracellular neutrophils, which predisposes patients with periodontitis and COVID-19 to more serious adverse effects and may cause constant tissue damage, in addition to the activation of autoimmune mechanisms ${ }^{31,32}$.

The elevation in blood levels of Markers D-dimer and C-reactive protein (CRP) has been reported in cases of patients with COVID-19 and moderate to severe periodontitis. This panorama has been related to the worsening of the clinical picture of viral infection, however, it still needs further studies ${ }^{32}$.

Biosafety care is fundamental due to the ability of the virus to remain suspended in the air for long periods and may contaminate mucous membranes and airways27. In this scenario, it is essential that the dentist recognizes the possible oral lesions of the covid-19 and adopts biosafety measures in order to identify early and contain viral transmission.

\section{CONCLUSION}

It is essential to perform an intraoral examination in patients with a positive result for COVID-19 or who are under suspicion of the disease, as this may be useful in the early detection of oral manifestations.

The knowledge of what has already been described in the literature facilitates the dentist in clinical decision making and influences the best prognosis for the patient. Also, they direct health professionals to adopt the appropriate biosafety measures, since they have a high risk of contamination.

Certainly, the detection of oral manifestations by the dentist can help in the early diagnosis of COVID-19, which reinforces the importance of the dentist in the pandemic.

It is notorious how is important to analyze whether the oral manifestations of COVID-19 are related to SARS-CoV-2 infection or if they are systemic consequences resulting from the impaired immune system, co-infection, or adverse reactions to medical treatment.

In this sense, further studies are needed in the scope of etiology and factors predisposing to the appearance of oral lesions resulting from this viral infection.

\section{REFERENCES}

1. Vieira AR. Oral manifestations in coronavirus disease 2019 (COVID-19). Oral Dis. 2020.

2. Sarode SC, Sarode GS, Sengupta N, Kumar Sharma N, Patil S. Biological behavior of oral squamous cell carcinoma in the background of novel corona virus infection. Oral Oncol. 2020 
3. Brandão TB, Gueiros LA, Melo TS. Oral lesions in patients with SARS-CoV-2 infection: could the oral cavity be a target organ? Oral Surgery, Oral Medicine, Oral Pathology and Oral Radiology. 2020 Aug.

4. Freni F, Meduri A, Gazia F, Nicastro V, Galletti C, Aragona P, et al. Symptomatology in head and neck district in coronavirus disease (COVID-19): A possible neuroinvasive action of SARS-CoV-2. Am J Otolaryngol. 2020; 41(5): 102612.

5. Casas CG, Català A, Hernández GC, Rodríguez-Jiménez P, Fernández-Nieto D, Lario AR, et al. Classification of the cutaneous manifestations of COVID-19: a rapid prospective nationwide consensus study in Spain with 375 cases. Br J Dermatol 2020;183(1):71-77

6. Carreras-Presas CM, Sánchez JA, López-Sánchez AF, Jané-Salas E, Pérez, MLS. Oral vesiculobullous lesions associated with SARS-CoV-2 infection. Oral Dis. 2020; 5: 1- 3.

7. Santos JAD, Normando AGC, Silva RLC, Acevedo AC, Canto GDL, Sugaya N, Santos-Silva AR, Guerra ENS. Oral Manifestations in Patients with COVID-19: A Living Systematic Review. J Dent Res. 2021;100(2): 141-154.

8. Rodríguez MD, Romera Aj, Villarroel M. Oral manifestations associated to Covid-19. Oral Diseases. 2020

9. Bodard, Anne-Gaëlle Deneuve, Sophie, Desoutter, Aline. Oral manifestation of Covid-19 as an inaugural symptom? Journal of Oral Medicine and Oral Surgery. 2020; 26:18

10. Dziedzic A, Wojtyczka R. The impact of coronavirus infectious disease 19 (COVID-19) on oral health. Oral Dis. 2020

11. Santos JA, Normando AGC, Silva RLC, Paula RM, Cembranel AC, Santos-Silva AR, Guerra ENS, Oral mucosal lesions in a COVID-19 patient: New signs or secondary manifestations? International Journal of Infectious Diseases. 2020; 97:326-328.

12. Rocha BA, Souto GR, Grossmann SMC, de Aguiar MCF, de Andrade BAB, Romañach $\mathrm{MJ}$, et al. Viral enanthema in oral mucosa: A possible diagnostic challenge in the COVID-19 pandemic. Oral Dis. Oral Dis. 2020

13. Herrera D, Serrano J, Roldán S, Sanz M. Is the oral cavity relevant in SARS-CoV-2 pandemic? Clinical Oral Investigations. 2020; 24(8): 2925-2930.

14. Kitakawa D, Oliveira FE, Castro PN, Carvalho LFCS. Short report - Herpes simplex lesion in the lip semimucosa in a COVID-19 patient. Eur Rev Med Pharmacol Sci. 2020; 24(17):9151-9153.

15. Ferreira ACAF, Romão TT, Macedo YS, Pupe C, Nascimento OJM. COVID-19 and herpes zoster co-infection presenting with trigeminal neuropathy. Eur J Neurol. 2020; 27(9):17481750 .

16. Sinadinos A, Shelswell J. Oral ulceration and blistering in patients with COVID-19. Evid Based Dent. 2020; 21(2): 49.

17. Jimenez-Cauhe J, Ortega-Quijano D, Carretero-Barrio I, Suarez-Valle A, Saceda-Corralo D, Real CMD. Erythema multiforme-like eruption in patients with COVID-19 infection: clinical and histological findings. Clin Exp Dermatol. 2020; 45(7):892-895.

18. Xiang Z, Koo H, Chen Q, Zhou X, Liu Y, Simon-Soro A. Potential implications of SARS-CoV-2 oral infection in the host microbiota. J Oral Microbiol. 2020; 13(1):1853451.

19. Lechien JR, Chetrit A, Chekkoury-Idrissi Y, Distinguin L, Circiu M, Saussez S, et al. Parotitis-Like Symptoms Associated with COVID-19, France, March-April 2020. Emerg Infect Dis. 2020; 26(9):2270-2271.
20. Hsu PJ, Yan K, Shi H, Izumchenko E, Agrawal N. Molecular biology of oral cavity squamous cell carcinoma. Oral Oncol 2020;102:104552.

21. Chern A, Famuyide AO, Moonis G. Sialadenitis: a possible early manifestation of COVID -19. Laryngoscope. 2020;130:25952597

22. Cai G, Bossé Y, Xiao F, Kheradmand F, Amos CI. Tobacco smoking increases the lung gene expression of ACE2, the receptor of SARS-CoV-2. Am J Respir Crit Care Med. 2020; 201(12):1557-9.

23. Martelli-Júnior H, Machado RA, Martelli DRB, Andrade MC, Coletta RD. Oral cancer and ACE2 receptor of SARS-CoV-2. Oral Oncol. 2020; 108:104920.

24. Sarode SC, Sarode GS, Sengupta N, Kumar Sharma N, Patil S. Biological behavior of oral squamous cell carcinoma in the background of novel coronavirus infection. Oral Oncol. 2020;110:104781.

25. Hinsley EE, Oliveira CE, Hunt S, Coletta RD, Lambert DW. Angiotensin 1-7 inhibits angiotensin II-stimulated head and neck cancer progression. Eur J Oral Sci 2017;125(4):247-57.

26. Warabi Y, Tobisawa S, Kawazoe T, Murayama A, Norioka $\mathrm{R}$, Morishima R, et al. Effects of oral care on prolonged viral shedding in coronavirus disease 2019 (COVID-19). Spec Care Dentist. 2020;40(5):470-474.

27. Wyllie AL, Fournier J, Casanovas-Massana A. Saliva is more sensitive for SARS-CoV-2 detection in COVID-19 patients than nasopharyngeal swabs. medRxiv. 2020.

28. Riad A, Kassem I, Issa J, Badrah M, Klugar M. Angular cheilitis of COVID-19 patients: A case-series and literature review. Oral Dis. 2020

29. Sinjari B, D’Ardes D, Santilli M, Rexhepi I, D'Addazio G, Di Carlo P, et al. SARS-CoV-2 and Oral Manifestation: An Observational, Human Study. J Clin Med. 2020; 9(10): 3218.

30. Freni F, Meduri A, Gazia F, Nicastro V, Galletti C, Aragona P, et al. Symptomatology in head and neck district in coronavirus disease (COVID-19): A possible neuroinvasive action of SARS-CoV-2. Am J Otolaryngol. 2020; 41(5): 102612.

31. Gupta S, Sahni V. The intriguing commonality of NETosis between COVID-19 \& Periodontal disease. Med Hypotheses 2020; 144: 109968.

32. Marouf N, Cai W, Said KN, Daas H, Diab H, Chinta VR, et al. Association between periodontitis and severity of COVID-19 infection: A case-control study. J Clin Periodontol. 2021;

33. Nuno-Gonzalez A, Martin-Carrillo P, Magaletsky K, Rios MDM, Mañas CH, Almazan JA, et al. Prevalence of mucocutaneous manifestations in 666 patients with COVID-19 in a field hospital in Spain: oral and palmoplantar findings. Br J Dermatol. 2021;184(1): 184-185.

34. Biadsee A, Biadsee A, Kassem F, Dagan O, Masarwa S, Ormianer Z. Olfactory and Oral Manifestations of COVID-19: Sex-Related Symptoms-A Potential Pathway to Early Diagnosis. Otolaryngol Head Neck Surg. 2020;163(4):722-728.

35. Hjelmesæth J, Skaare D. Loss of smell or taste as the only symptom of COVID-19. Tidsskr Nor Laegeforen. 2020; 3: 140(7)

36. Melley LE, Bress E, Polan E. Hypogeusia as the initial presenting symptom of COVID-19. BMJ Case Rep. 2020;13(5):e236080.

37. Malayala SV, Raza A. A Case of COVID-19-Induced Vestibular Neuritis. Cureus. 2020;12(6):8918. 
38. Moreira MS, Neves ILI, Bernoche CYSM, Sarra G, Santos-Paul MA, Silva FCN, Schroter GT, et al. Bilateral paresthesia associated with cardiovascular disease and COVID-19. Oral Dis. 2020; 8

39. Riad A, Kassem I, Badrah M, Klugar M. Acute parotitis as a presentation of COVID-19? Oral Diseases. 2020

40. Halboub E, Al-Maweri SA, Alanazi RH, Qaid NM, Abdulrab S. Orofacial manifestations of COVID-19: a brief review of the published literature. Braz Oral Res. 2020; 34:e124.
41. Soares CD, Mosqueda-Taylor A, Carvalho MGF, Almeida OP Oral vesiculobullous lesions as an early sign of COVID-19: immunohistochemical detection of SARS-CoV-2 spike protein. Br J Dermatol. 2021;184(1):e6.

42. Thomas DC, Baddireddy SM, Kohli D. Anosmia: A review in the context of coronavirus disease 2019 and orofacial pain. J Am Dent Assoc. 2020; 151(9):696-702. 